\title{
Analysis of Unsteady Hydromagnetic Natural Convective Dissipative Flow through an Inclined Plate
}

\author{
J. F. Baiyeri ${ }^{1}$, O. A. Esan ${ }^{1}$, T. O. Ogunbayo ${ }^{1}$, O. E. Enobabor ${ }^{1}$ and S. O. Salawu ${ }^{2^{\star}}$ \\ ${ }^{1}$ Department of Mathematics, Yaba College of Technology, Yaba, Nigeria. \\ ${ }^{2}$ Department of Mathematics, University of Ilorin, Ilorin, Nigeria.
}

Authors' contributions

This work was carried out in collaboration between all authors. Author JFB formulated the problem and the derivation of equations, author OAE solved the problem, did the table and the graphs. Author

SOS carried out the mathematical analysis and discussion of result while authors TOO and OEE managed the literature searches and review. All authors read and approved the final manuscript.

Article Information

DOI: $10.9734 / A J O P A C S / 2017 / 35008$

Editor(s):

(1) Stanislav Fisenko, Department of Mathematics, MSLU, Russia.

Reviewers:

(1) B. Prabhakar Reddy, The University of Dodoma, Tanzania.

(2) P. A. Murad, Morningstar Applied Physics Inc., LLC, Austria. Complete Peer review History: http://www.sciencedomain.org/review-history/20496

Original Research Article

Received $23^{\text {rd }}$ June 2017

Accepted $13^{\text {th }}$ July 2017

Published $14^{\text {th }}$ August 2017

\begin{abstract}
This study investigates the effects of viscous dissipation on an unsteady two-dimensional magnetohydrodynamics (MHD) natural convection through an inclined plate. The governing partial differential equations together with the boundary conditions are transformed into a dimensionless coupled partial differential equations. An implicit-finite difference method is use to solve the dimensionless equations. The effects of various fluid parameters on velocity, temperature, concentration distribution are separately presented in graphical forms and discussed. It was observed that the an increase in the thermal Grashof number or modify Grashof number is manifested as an increase in flow velocity also magnetic field and viscous dissipation is seen to exert a more significant effect on the flow field and thus on the heat transfer from the plate to the fluid.
\end{abstract}

Keywords: Viscous dissipation; MHD; natural convection; inclined plate; finite difference. 


\section{INTRODUCTION}

Dissipation of energy is significant when considering the unsteady MHD natural convective flow through an inclined plate. Viscous dissipation effect is reflected by the Eckert number which is a source of the temperature rise that takes place in the flow of the fluid. Magnetohydrodynamics has an important application in several engineering problems such as MHD power generators in the boundary layer control aerodynamics, nuclear reactors cooling and also in the petroleum industries.

In view of its application, Mukhopadhyay et al. [1] studied MHD boundary layer flow over a heated stretching sheet with variable viscosity. Gnaneswara [2] carried out Lie group analysis of heat and mass transfer effects on steady MHD free convection dissipative fluid flow past an inclined porous surface with heat generation. Gnaneswara and Bhaskar [3] investigated mass and heat generation effects on MHD free convection flow past inclined vertical surface in a porous medium while Saidul [4] carried out analysis on the MHD free convection and mass transfer flow past through an inclined plate with heat generation. The boundary layer equation in two-dimensional energy and mass transfer equations were obtained by boundary layer approximation. It was shown that the effects of Magnetic parameter and Heat source parameter enhanced the velocity field. Kalpadides and Balassas [5] studied the free convective boundary layer problem of an electrically conducting fluid over an elastic surface by group theoretic method. It was found from the numerical solution that the effect of increasing thermal Grashof number is manifested by an increase in flow velocity. In the presence of a magnetic field parameter, the permeability of porous medium, viscous dissipation is demonstrated to exert a more significant effect on the flow field and thus, on the heat transfer from the plate to the fluid. The velocity and concentration is found to decrease gradually as the Schmidtl number is increased.

The study of flow through porous medium and dissipation effect has received attention of many researchers because of it extensive applications in enhancing recovery of petroleum, chemical engineering etc. Sandeep and Sugunamma [6] analysed the effect of inclined magnetic field on unsteady free convective flow of dissipative flow past a vertical plate. Dada and Adefolaju [7] investigated dissipation, MHD and radiation effects on an unsteady convective heat and mass transfer in a Darcy-Forcheimer porous medium. It was noted that temperature and concentration slightly increase with increase in magnetic field parameter while the presence of magnetic field has a retarding effect on the velocity profile. A rise in the conduction-radiation parameter causes reduction in the velocity profile while a rise in the dissipation function induces a considerable rise in velocity. Increase in the Prandtl number, Schmidtl number, thermal Grashof number, solutant Grashof number and the conduction radiation parameter causes the temperature to reduce while a rise in the Darcy number causes a rise in temperature along and normal to the wall. Hunegnaw and Kishan [8] carried out analysis on unsteady MHD heat and mass transfer flow over stretching sheet in porous medium with variable properties considering viscous dissipation and chemical reaction while Megahed et al. [9] Studied a similarity analysis in magnetohydrodynamics hall effects on free convection flow and mass transfer past a semi-infinite vertical flat plate. Ibrahim [10] investigated the similarity reductions for problems of radiative and magnetic field effects on free convection and mass-transfer flow past a semi-finite flat plate. They obtained new similarity reductions and found an analytic solution for the uniform magnetic field by using Lie group method. Chen [11] carried out an analysis to study the natural convection flow over a permeable inclined surface with variable wall temperature and concentration. The result show that the velocity is decreased in the presence of a magnetic field. Increasing the angle of inclination decreases the effect of buoyancy force. Heat transfer rate is increased when the Prandtl number is increased. Reddy and Reddy [12] performed an analysis to study the natural convection flow over a permeable inclined surface with variable temperature, momentum and concentration.

Follow from the above studied, the present study investigate the effects of viscous dissipation on MHD free convection and mass transfer flow with heat generation through an inclined plate.

\section{PROBLEM FORMULATION}

Considering an unsteady two-dimensional MHD natural convection and dissipating fluid past an inclined plate in a Cartesian coordinate system where the $X$-axis is chosen along the plate in the direction of the flow and the $\mathrm{Y}$-axis is normal to it. It has been considered initially that the plate as 
well as the fluid is at the same temperature $T_{\infty}$ and the concentration levels $C_{\infty}$ everywhere in the fluid. Also, it is considered that the fluid and the plate is at rest after the plate is to be moving with a constant velocity $U_{0}$ in its own plane and instantaneously at time $t>0$, the concentration and the temperature of the plate are raised to
$C_{w}>C_{\infty}$ and $T_{w}>T_{\infty}$ respectively, where $C_{w}$, $T_{w}$ are concentration and temperature at the wall of the plate respectively and $C_{\infty}, T_{\infty}$ are the concentration and temperature far away from the plate respectively. The physical model of the problem is shown in the Fig. 1 below.

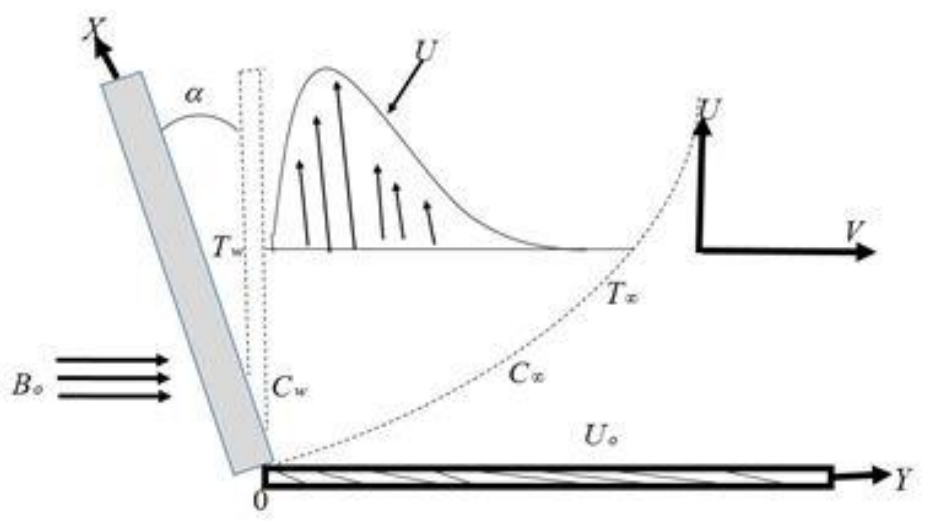

Fig. 1. The physical model and coordinate system

With reference to the generalized governing equations described above, the transient two dimensional problems are governed by the following system of coupled non-linear differential equations.

$$
\begin{aligned}
& \frac{\partial u}{\partial x}+\frac{\partial v}{\partial y}=0 \\
& \frac{\partial u}{\partial t}+u \frac{\partial u}{\partial x}+v \frac{\partial u}{\partial y}=v \frac{\partial^{2} u}{\partial y^{2}}+g \beta\left(T-T_{\infty}\right) \cos \alpha+g \beta^{*}\left(C-C_{\infty}\right) \cos \alpha-\frac{\sigma \beta_{0}^{2}}{\rho} u \\
& \frac{\partial T}{\partial t}+u \frac{\partial T}{\partial x}+v \frac{\partial T}{\partial y}=K_{T} \frac{\partial^{2} T}{\partial y^{2}}+\frac{Q_{T}}{\rho C_{p}}+\frac{\mu}{\rho C_{p}}\left(\frac{\partial u}{\partial y}\right)^{2} \\
& \frac{\partial C}{\partial t}+u \frac{\partial C}{\partial x}+v \frac{\partial C}{\partial y}=D \frac{\partial^{2} C}{\partial y^{2}}
\end{aligned}
$$

The corresponding initial and boundary conditions are:

$$
\text { at } \begin{array}{rrrrr}
t & =0, & u & =0, \quad v=0, & C \rightarrow C_{\infty}, \text { everywhere } \\
u & =0, \quad v=0, \quad T \rightarrow T_{\infty}, & C \rightarrow C_{\infty} \text { at } x=0
\end{array}
$$




$$
\begin{aligned}
& \text { at } t>0 \quad u=U_{0}, \quad v=0, T \rightarrow T_{\infty}, \quad C \rightarrow C_{\infty} \text { at } \quad y=0 \\
& \left.u=0, \quad v=0, \quad T \rightarrow T_{w}, \quad C \rightarrow C_{w} \text { as } \quad y \rightarrow \infty\right\}
\end{aligned}
$$

where $x, y$ are Cartesian coordinate system, $u, v$ are $x, y$ component of flow velocity respectively. Here $\mathrm{g}$ is the local acceleration due to gravity; $\mu$ is the dynamic viscosity; $\rho$ is the density of the fluid; $K$ is the thermal conductivity, $C_{p}$ is the specific heat at the constant pressure; $D$ is the coefficient of mass diffusivity; $\beta$ and $\beta^{*}$ are the thermal and concentration expansion coefficients respectively; $\alpha$ is the angle of inclination; $T$ is the temperature of the fluid; $\sigma$ is the electrical conductivity of the fluid; $T_{\infty}$ is the temperature of the fluid far away from the plate; $\mathrm{C}$ is the fluid concentration; $C_{\infty}$ is the concentration in the fluid far away from the surface; $B_{0}$ is the magnetic induction and $v$ is the kinematic viscosity.

Introducing the following dimensionless variables;

$$
X=\frac{x U_{0}}{v} ; Y=\frac{y U_{0}}{v} ; U=\frac{u}{U_{0}} ; V=\frac{v}{U_{0}} ; \tau=\frac{t U_{0}^{2}}{v} ; \bar{T}=\frac{T-T_{\infty}}{T_{w}-T_{\infty}} ; \bar{C}=\frac{C-C_{\infty}}{C_{w}-C_{\infty}} ; Q^{*}=\frac{Q_{T}}{\left(T-T_{\infty}\right)}(7)
$$

where $\mathrm{X}$ and $\mathrm{Y}$ are dimensionless coordinates, $\mathrm{U}$ and $\mathrm{V}$ are dimensionless velocities, $\tau$ is the dimensionless time, $\bar{T}$ is the dimensionless temperature function, $\bar{C}$ is the dimensionless concentration function. Applying these dimensionless variables to equations (1) - (4) reduces to the non dimensional equations:

$$
\begin{aligned}
& \frac{\partial U}{\partial X}+\frac{\partial V}{\partial Y}=0 \\
& \frac{\partial U}{\partial \tau}+U \frac{\partial U}{\partial X}+V \frac{\partial U}{\partial Y}=\frac{\partial^{2} U}{\partial Y^{2}}+G r \bar{T} \cos \alpha+G m \bar{C} \cos \alpha-M U \\
& \frac{\partial \bar{T}}{\partial \tau}+U \frac{\partial T}{\partial X}+V \frac{\partial T}{\partial y}=\frac{1}{P r} \frac{\partial^{2} \bar{T}}{\partial y^{2}}+\bar{T} \cdot \gamma+E_{c}\left(\frac{\partial U}{\partial y}\right)^{2} \ldots \ldots \ldots \ldots \ldots \ldots \ldots \ldots \ldots \ldots \\
& \frac{\partial \bar{C}}{\partial \tau}+U \frac{\partial \bar{C}}{\partial X}+V \frac{\partial \bar{C}}{\partial Y}=D \frac{\partial^{2} \bar{C}}{\partial Y^{2}}
\end{aligned}
$$

where $\frac{v g \beta\left(T_{w}-T_{\infty}\right)}{U_{\circ}^{3}}=G r=$ GrashofNumber, $\frac{v g \beta^{*}\left(C_{w}-C_{\infty}\right)}{U_{\circ}^{3}}=G m=$ ModifiedGrashofNumber, $\frac{\sigma \beta_{\circ}^{2} V}{\rho U_{\circ}^{2}}=M=$ MagneticParameter, $\operatorname{Pr}=\frac{V C_{p}}{k}=$ Prandltnumber, $\quad S c=\frac{V}{D}=$ Schmidtlnumber,

$$
E_{c}=\frac{\mu U_{0}^{2}}{C_{p}\left(T_{w}-T_{\infty}\right) V}=\text { Eckertnumber, } \gamma=\frac{V Q^{*}}{U_{\circ}^{2} \rho C_{p}}=\text { Heatsourceparameter }
$$

Also the associated initial and boundary conditions becomes 


$$
\left.\begin{array}{clllll}
\tau=0 & U=0, \quad V=0 & \bar{T}=0 & \bar{C}=0 & \text { everywhere } \\
& U=0, \quad V=0 & \bar{T}=0 & \bar{C}=0 & \text { atX }=0
\end{array}\right\}
$$

\section{NUMERICAL SOLUTION OF THE PROBLEM}

Solving the unsteady non-linear coupled partial differential equations (8) - (11) subject to the conditions given by (12) and (13) and employ an implicit finite difference of the Crank-Nicolson type. The numerical algorithm presented in this article is conjectured to work for any value of the time step! The discretization of the governing equations is based on a linear Cartesian mesh and uniform grids on which finite-differences are taken; these are first-order accurate in time but second order in space, which improves the accuracy in time to second order. The coupled non-linear partial differential are converted to a difference equation. We define the coordinate $(X, Y, \tau)$ of the mesh points of the solution domain by $X=i \Delta X, Y=j \Delta Y$ and $\tau=k \Delta \tau$ where $i, j, k$ are positive integers and we denote the values of $U$ at the mesh points by $U(i \Delta x, j \Delta y, k \Delta \tau)=U_{i, j}^{k}$ and $V(i \Delta x, j \Delta y$, $k \Delta \tau)=V_{i, j}^{k}$. The finite difference equations corresponding to the equations are given as follows:

$$
\begin{aligned}
& \frac{\left[U_{i, j}^{k+1}-U_{i-1, j}^{k+1}+U_{i, j}^{k}-U_{i-1, j}^{k}+U_{i, j-1}^{k+1}-U_{i-1, j-1}^{k+1}+U_{i, j-1}^{k}-U_{i-1, j-1}^{k}\right]}{4 \Delta X}+ \\
& \frac{\left[V_{i, j}^{k+1}-V_{i, j-1}^{k+1}+V_{i, j}^{k}-V_{i, j-1}^{k}\right]}{2 \Delta Y}=0 \\
& \frac{\left[U_{i, j}^{k+1}-U_{i, j}^{k}\right]}{\Delta \tau}+U_{i, j}^{k} \frac{\left[U_{i, j}^{k+1}-U_{i-1, j}^{k+1}+U_{i, j}^{k}-U_{i-1, j}^{k}\right]}{2 \Delta X}+ \\
& V_{i, j}^{k} \frac{\left[U_{i, j+1}^{k+1}-U_{i, j-1}^{k+1}+U_{i, j+1}^{k}-U_{i, j-1}^{k}\right]}{4 \Delta Y}= \\
& \frac{\left[U_{i, j-1}^{k+1}-2 U_{i, j}^{k+1}+U_{i, j+1}^{k+1}+U_{i, j-1}^{k}-2 U_{i, j}^{k}+U_{i, j+1}^{k}\right]_{+}}{2(\Delta Y)^{2}}+ \\
& G_{r} \frac{\left[T_{i, j}^{k+1}+T_{i, j}^{k}\right]}{2} \cos \alpha+G_{m} \frac{\left[C_{i, j}^{k+1}+C_{i, j}^{k}\right]}{2} \cos \alpha-M \frac{\left[U_{i, j}^{k+1}+U_{i, j}^{k}\right]}{2} \\
& \frac{\left|\bar{T}_{i, j}^{k+1}-\bar{T}_{i, j}^{k}\right|}{\Delta \tau}+U_{i, j}^{k} \frac{\left|\bar{T}_{i, j}^{k+1}-\bar{T}_{i-1, j}^{k+1}+\bar{T}_{i, j}^{k}-\bar{T}_{i-1, j}^{k}\right|}{2 \Delta X_{1}} \\
& +V_{i, j}^{k} \frac{\left.\Delta_{[i, j+1}^{\tau}-\bar{T}_{i, j-1}^{k+1}+\bar{T}_{i, j+1}^{k}-\bar{T}_{i, j-1}^{k}\right]_{i}^{k+1}}{4 \Delta Y}= \\
& \frac{1}{P_{r}} \frac{\left[\bar{T}_{i, j-1}^{k+1}-2 \bar{T}_{i, j}^{k+1}+\bar{T}_{i, j+1}^{4+1}+\bar{T}_{i, j-1}^{k}-2 \bar{T}_{i, j}^{k}+\bar{T}_{i, j+1}^{k}\right]}{2(\Delta Y)^{2}} \\
& +\gamma \frac{\left[T_{i, j}^{k+1}+T_{i, j}^{k}\right]}{2}+E_{c}\left[\frac{U_{i, j+1}^{k}-U_{i, j}^{k}}{2 \Delta Y}\right]^{2}
\end{aligned}
$$




$$
\begin{aligned}
& \frac{\left|\bar{C}_{i, j}^{k+1}-\bar{C}_{i, j}^{k}\right|_{+} U_{i, j}^{k}}{\Delta \tau} \frac{\left.\mid \bar{C}_{i, j}^{k+1}-\bar{C}_{i-1, j}^{k+1}+\bar{C}_{i, j}^{k}-\bar{C}_{i-1, j}^{k}\right\rfloor_{+}}{2 \Delta_{y} X} \\
& V_{i, j}^{k} \frac{\left[\bar{C}_{i, j+1}^{k+1}-\bar{C}_{i, j-1}^{k+1}+\bar{C}_{i, j+1}^{k}-\bar{C}_{i, j-1}^{k} \Delta_{y} Y\right.}{4 \Delta Y}= \\
& \frac{1}{S_{c}} \frac{\left[\bar{C}_{i, j-1}^{k+1}-2 \bar{C}_{i, j}^{k+1}+\bar{C}_{i, j+1}^{k+1}+\bar{C}_{i, j-1}^{k}-2 \bar{C}_{i, j}^{k}+\bar{C}_{i, j+1}^{k}\right]}{2(\Delta Y)^{2}}
\end{aligned}
$$

The region of consideration here is a rectangle with sides $X_{(\max )}$ and $Y_{(\max )}$. Where $X_{(\max )}$ corresponds to $Y=\infty$ which lies outside the momentum, thermal and concentration boundary layers. The subscripts $i$ and $j$ denotes the grid points with $X$ and $Y$ coordinates respectively and $\mathrm{k}$ along the t-direction. Dividing $\mathrm{X}$ and $\mathrm{Y}$ into $\mathrm{M}$ and $\mathrm{N}$ grid spacing respectively, the mesh sizes are taken as $\Delta X=0.05$ and $\Delta Y=0.05$ and $\Delta t=0.001$. During any one-time step, the coefficients $U_{i, j}^{k}$ and $V_{i, j}^{k}$ appearing in the difference equations are treated as constants. The values of $\mathrm{C}, \mathrm{T}, \mathrm{U}$ and $\mathrm{V}$ are known at all grid point at $t=0$ from the boundary/initial conditions. The values of $\mathrm{C}, \mathrm{T}, \mathrm{U}$ and $\mathrm{V}$ at time level $(k+1)$ are evaluated using the already known values at previous time level (k).

Hence, the finite difference equation forms a tridiagonal system of equations at every internal nodal point on a particular $i$-level which is solved by using Matlab programming package that employ Thomas Algorithm as discussed Carnahan et al. [13]. Thus we calculate the values of $C$ and $T$ at every nodal point for a particular $i$ at $(k+1)$ th time level and the results obtained were used in $U$ at $(k+1)$ th time level. The values of $\mathrm{V}$ are also determined at every nodal point explicitly on a particular $i$-level at $(k+1)$ th time level. In this way, the values of $C, T, U$ and $V$ are obtained at all grid point at time level $(k+1)$ th in the region. The process is repeated several times for various $i$-level until the steady state is reached which is assumed to have been reached when the absolute difference between the values of $U, T$ and $C$ at two consecutive time steps are less than $10^{-5}$ at all grid points.

\section{DISCUSSION OF RESULTS}

Numerical computations has been carried out using the method of implicit finite difference of the Crank-Nicolson type for variations in the fluid's parameters, namely the thermal Grashof number $\mathrm{Gr}$, solutal Grashof number Gm, magnetic field parameter $M$, heat source parameter $\gamma$ angle of inclination $\alpha$, prandtl number $\mathrm{Pr}$, eckert number Ec and schmidtl number Sc. The solutions for the velocity $U$ versus $\mathrm{Y}$, temperature $\bar{T}$ versus $\mathrm{Y}$, concentration $\bar{C}$ versus $\mathrm{Y}$ are shown in the graphs for different values of parameter.

Fig. 2 shows the effect of angle of inclination to the vertical direction on the velocity profile for various angles of inclination $\left(\alpha=0^{\circ}, 30^{\circ}, 45^{\circ}, 60^{\circ}\right)$ with fixed values $P_{r}=0.71, S_{c}=0.6, M=0.5, G_{r}=20, G_{m}=20$, $\gamma=0.2$. From this figure, it is observed that the velocity is decreased by increasing the angle of inclination. The fact is that as the angle of inclination increases the effect of buoyancy force due to thermal diffusion decreases by a factor of $\cos \alpha$.

Fig. 3 represents the velocity distributions for different values of magnetic parameter $(M=0.5,2.5,4.5)$ the values of $P_{r}=0.71, S_{c}=0.6, G_{r}=20, G_{m}=20 . \gamma=0.2$, are constants and with an inclination angle $0^{\circ}$. In this figure, it is observed that velocity distribution decreases with an increase in magnetic parameter. Since magntic field exact a retarding force on fluid flow.

Fig. 4 depicts the velocity distribution for different values of Thermal Grashof number $\left(G_{r}=20,30,40\right)$ and the values of $P_{r}=0.71, S_{c}=0.6, M=0.5, G_{m}=20, \gamma=0.2$,

are fixed and with an inclination angle $0^{\circ}$. The positive values of $G_{r}$ is correspond to the cooling of the plate. It is observed that velocity distribution increases with the increase of Grashof numbers. 


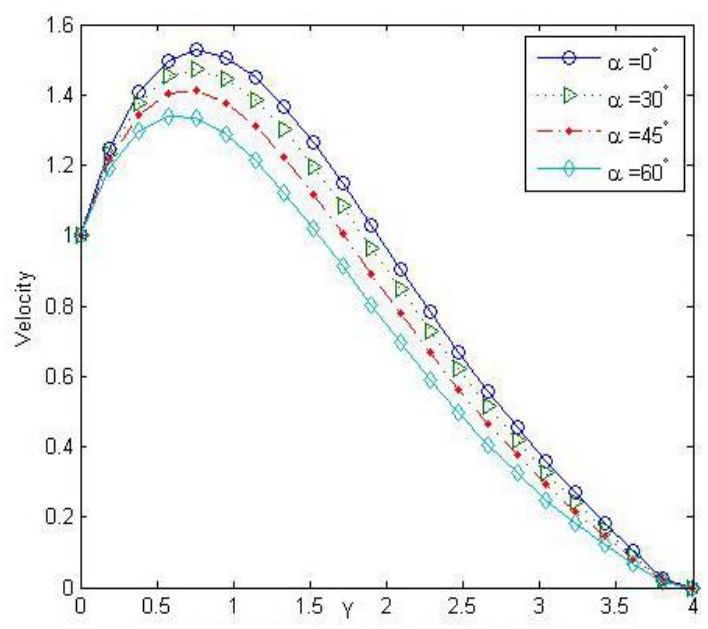

Fig. 2. Velocity profile for different values of $\alpha$

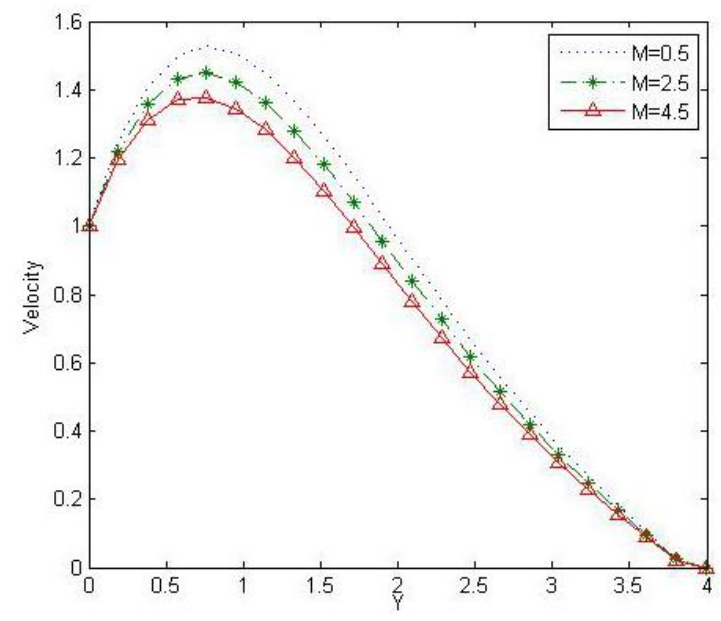

Fig. 3. Velocity profile for different values of $M$

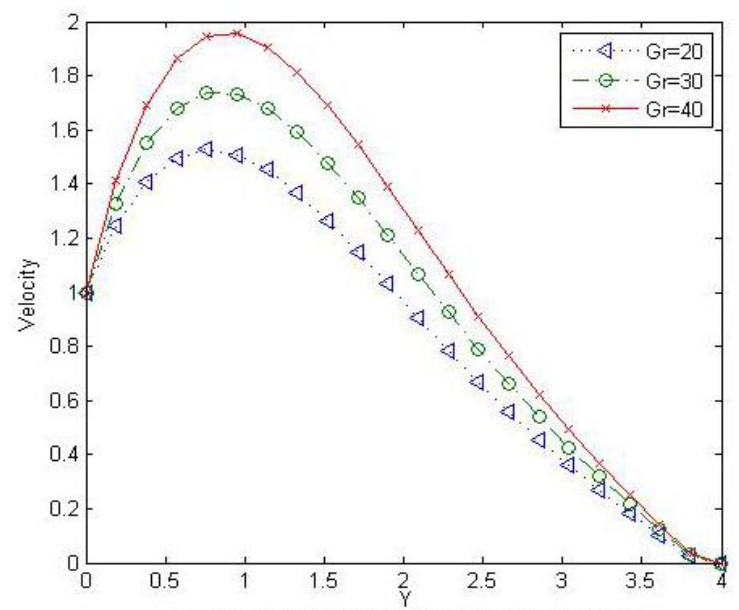

Fig. 4. Velocity profile for different values of $\mathrm{Gr}$ 
Fig. 5 shows the velocity distribution for different values of Prandtl number $\left(P_{r}=0.71,1.0,7.0\right)$ and the values of $M=0.5$, $G_{r}=20, G_{m}=20, S_{c}=0.6, \gamma=0.2 \quad$ are maintained with an inclination of $0^{\circ}$. The Prandtl number defines the ratio of momentum diffusivity to thermal diffusivity. The numerical results show that the effect of increasing values of Prandtl number results in a decreasing velocity.

Fig. 6 represents the velocity distribution for different values of Modify Grashof number $\left(G_{m}=20,30,40\right)$ and the values of $G_{r}=20, M=0.5, \gamma=0.2, S_{c}=0.60, P_{r}=0.71$ are constants with an inclined angle $0^{\circ}$. The solutant Grashof number $G_{m}$ defines the ratio of the species buoyancy force to the viscous hydrodynamic force. The velocity distribution increases with an increase in the solutant Grashof number.

Fig. 7 shows the effect of viscous dissipation parameter on the velocity distribution for different values of Eckert number Ec $=0.001,1.0,1.5,2.5$ and the values of $G_{r}=20, G_{m}=20, M=0.5, \gamma=0.2, S_{c}=0.60, P_{r}=0.71$ are kept unchanged with an inclined angle $0^{0}$. The Eckert number Ec expresses the relationship between the kinetic energy in the flow and enthalpy. It embodies the conversion of kinetic energy into internal energy by the workdone against the viscous fluid stresses. The positive

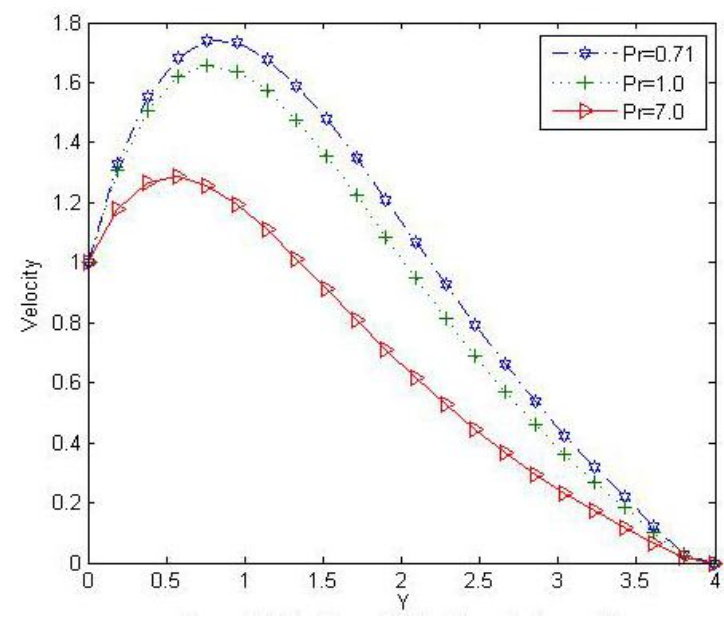

Fig. 5. Velocity profile for different values of $\mathrm{Pr}$

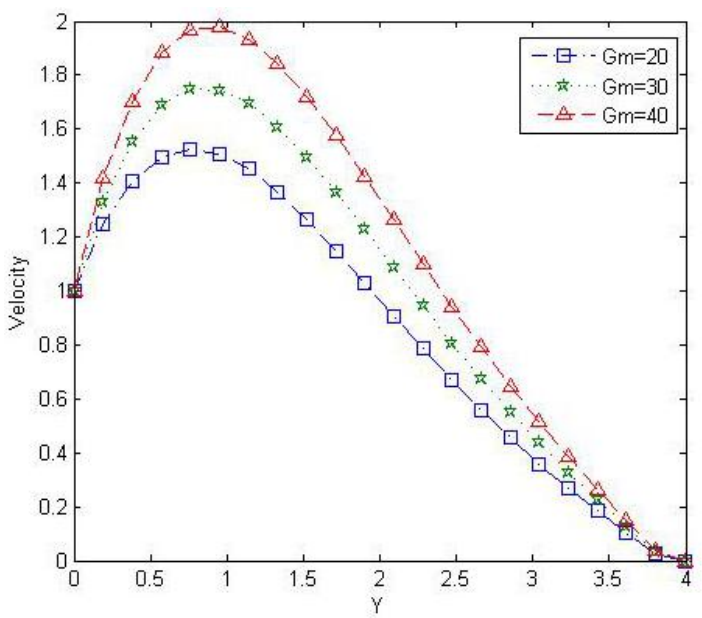

Fig. 6. Velocity profile for different values of Gm 
Eckert number indicates cooling of the plate. i.e loss of heat to the fluid from the plate. It was observed that the velocity distribution increase with the increases of Eckert number. Hence, higher viscous dissipative heat causes a rise in the velocity distribution.

Fig. 8 depicts the temperature distribution for different values of Heat Source parameter $(\gamma=0.5,1.5,2.5,3.5)$ and the values of $G_{r}=20, G_{m}=20, M=0.5, S_{c}=0.60, P_{r}=0.71$

are constants with an inclined angle $0^{0}$. In this figure, it is observed that temperature distribution increases with a rise in Heat source parameter.

Fig. 9 represents the temperature distribution for different values of Prandtl number $\left(P_{r}=0.45,1.0,3.0,5.0\right)$ and the values of
$G_{r}=20, G_{m}=20, M=0.5, \gamma=0.2, S_{c}=0.60$

are constants with an inclined angle $0^{\circ}$. The number defines the ratio of momentum diffusivity to thermal diffusivity. It is observe that for smaller values of $\mathrm{Pr}$, the thermal conductivities increased. It implies that heat is able to diffuse away from the heated plate faster than for higher values of $\operatorname{Pr}$.

Fig. 10 represents the Velocity profile for different values of Schmidtl number. $\left(S_{c}=0.6,0.78,0.94,1.0\right)$ and the values of $G_{r}=20, G_{m}=20, M=0.5, \gamma=0.2, P_{r}=0.71$ are constants with an inclined angle $0^{\circ}$.The Schmidtl number embodies the ratio of the momentum to the mass diffusivity. It is observed that increase in the Schmidtl number Sc decreases the velocity.

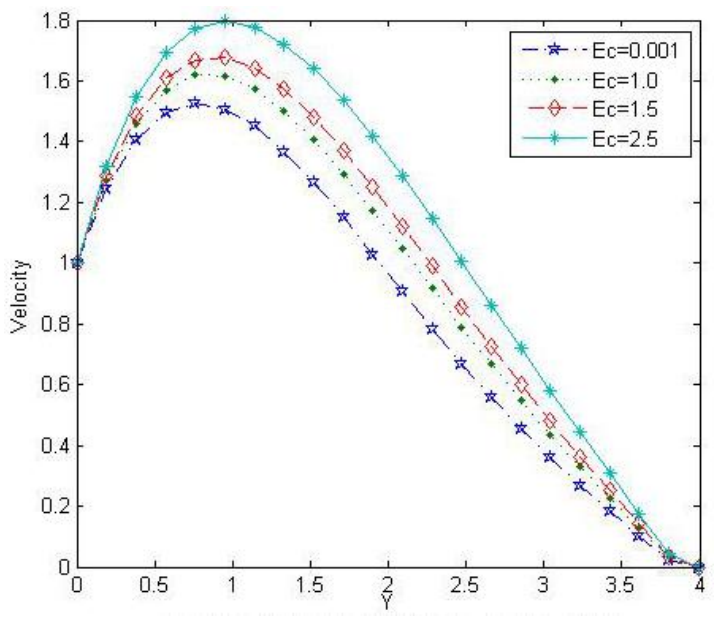

Fig. 7. Velocity profile for different values of Ec

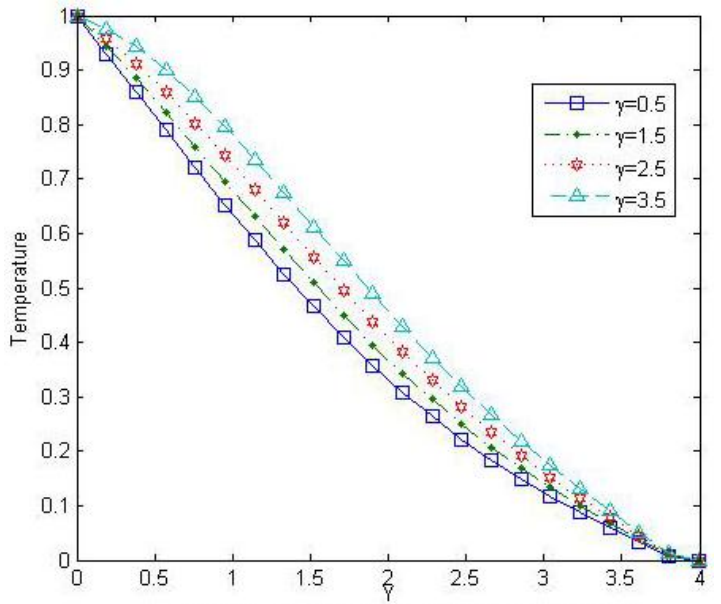

Fig. 8. Velocity profile for different values of $y$ 
Fig. 11 represents the Concentration profile for different values of Schmidtl number. $\left(S_{c}=0.6,0.78,0.94,1.0\right)$ and the values of $G_{r}=20, G_{m}=20, M=0.5, \gamma=0.2, P_{r}=0.71$ are constants with an inclined angle $0^{\circ}$. It is observed that increase in the Schmidtl number decreases the Concentration.

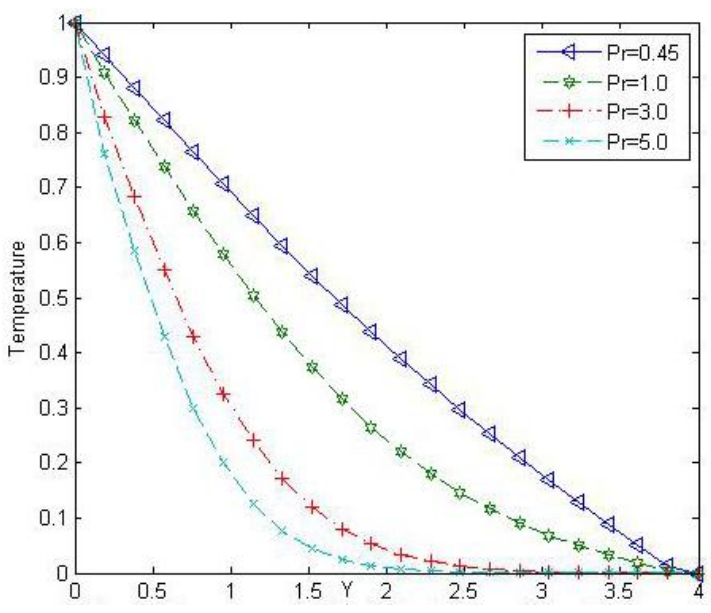

Fig. 9. Temperature distribution for different values of $\mathrm{Pr}$

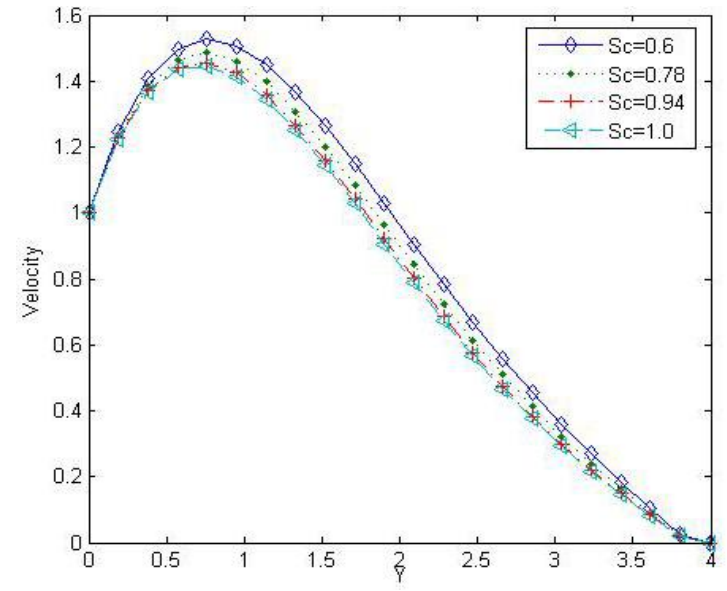

Fig. 10. Velocity profile for different values of Sc

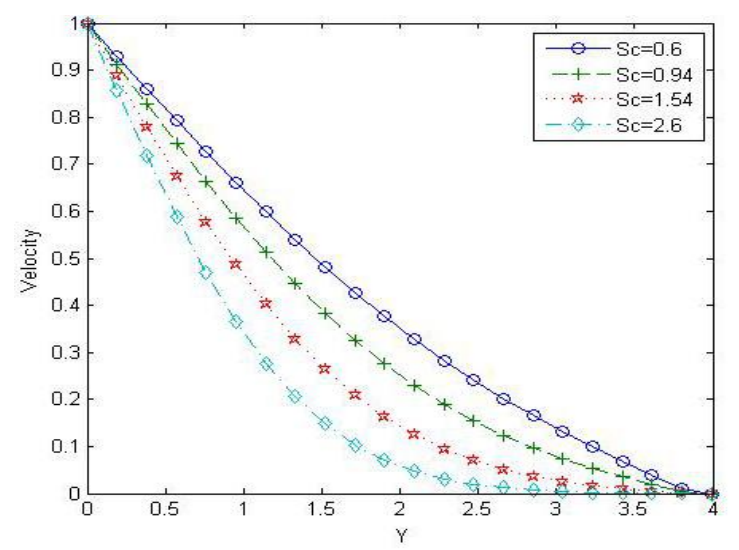

Fig. 11. Concentration profile for different values of Sc 


\section{CONCLUSION}

This study deals with the unsteady MHD natural convection dissipative flow through an inclined plate. The equations governing the model were highlighted and non-dimensionalized. The effect of the angle of inclination through the plate on the fluid flow was observed. The mathematical equations to the problem were then solved using Crank-Nicolson method. Series of computation was carried out to study graphically the effects of the controlling thermo-physical parameters which are Magnetic field $(M)$, dissipative function $\left(E_{c}\right)$, thermal Grashof number $\left(G_{r}\right)$, Modify Grashof number $\left(G_{m}\right)$, Prandtl number $\left(P_{r}\right)$, Schmidtl number $\left(S_{c}\right)$, Heat source parameter $(\gamma)$, and the angle of inclination $(\alpha)$ on the dimensionless velocity, temperature and concentration profiles.

It is also seen that the effect of increasing thermal Grashof number or Modify Grashof number is manifested as an increase in flow velocity. It is also interesting to note that the temperature decreases much faster than the air temperature. In the presence Magnetic field parameter, viscous dissipation is demonstrated to exert a more significant effect on the flow field and thus on the heat transfer from the plate to the fluid. The velocity and concentration is found to decrease gradually as the Schmidtl number is increased. The velocity and temperature distribution also increases with increases of the Heat source parameter.

\section{COMPETING INTERESTS}

Authors have declared that no competing interests exist.

\section{REFERENCES}

1. Mukhopadhyay S, Layek GC, Samad SA. Study of MHD boundary layer flow over a heated stretching sheet with variable viscosity. Int. J. Heat Mass Transfer. 2005;48:4460-4466.

2. Gnaneswara RM. Lie group analysis of heat and mass transfer effects on steady MHD free convection dissipative fluid flow past an inclined porous surface with heat generation. Journal of
Applied Fluid Mechanic. 2012;39(3):233254.

3. Gnaneswara RM, Bhaskar RN. Mass and heat generation effects on MHD free convection flow past inclined vertical surface in a porous medium. Journal of Applied Fluid Mechanic. 2011; 4(3):7-11.

4. Saidul Islam MD, Samsuzzoha MD, Ara S, Dey P. MHD free convection and mass transfer flow with heat generation through an inclined plate. Annals of Pure and Applied Mathematics. 2013; 3:129-141.

5. Kalpakides VK, Balassas KG. Symmetry groups and similarity solution for $a$ free convective boundary-layer problem. Int. J. Non-linear Mech. 2004;39:16591670.

6. Sandeep N, Sugunamma V. Effect of inclined magnetic field on unsteady free convective flow of dissipative flow past a vertical plate. Journal of Advanced Engineering Techniques. 2013; 1:6-23.

7. Dada MS, Adefolaju FH. Dissipation, MHD and radiation effects on an unsteady convective heat and mass transfer in a Darcy-Forcheimer porous medium. Journal of Mathematics Research. 2012;4(2):110127.

8. Hunegnaw D, Kishan N. Unsteady MHD heat and mass transfer flow over stretching sheet in porous medium with variable properties considering viscous dissipation and chemical reaction. American Chemical Science Journal. 2014;4(6):902-917.

9. Megahed AA, Komy SR, Afify AA. Similarity analysis in magnetohydrodynamics hall effects on free convection flow and mass transfer past a semi-infinite vertical flat plate. International Journal of Non-linear Mechanics. 2003;38: 513-520.

10. Ibrahim FS, Mansour MA, Hamad MA. Lie group analysis of radiative and magnetic field effects on free convection and mass transfer flow past a semi-infinite vertical flat plate. Electronic J. Differential Equations. 2005; 39:1-17.

11. Chen $\mathrm{CH}$. Heat and mass transfer in MHD flow by natural convection from a permeable, inclined surface with 
variable wall temperature and concentration. Acta Mechanica. 2004; 172:219-235.

12. Reddy MG, Reddy NB. Mass transfer and heat generation effects on MHD free convection flow past an inclined vertical surface in a porous medium. Journal of Applied Fluid Mechanics. 2011;4(1):7-11.

13. Carnahan B, Luther HA, Willkes JO. Applied Numerical Method. John Wiley and Sons, New York, USA; 1969.

(c) 2017 Baiyeri et al.; This is an Open Access article distributed under the terms of the Creative Commons Attribution License (http://creativecommons.org/licenses/by/4.0), which permits unrestricted use, distribution, and reproduction in any medium, provided the original work is properly cited.

Peer-review history:

The peer review history for this paper can be accessed here: http://sciencedomain.org/review-history/20496 